\title{
OPTIMIZATION OF THE ULTRASONIC PROCESSING IN A MELT FLOW
}

\author{
I Tzanakis ${ }^{1,2}$, G S B Lebon ${ }^{3}$, D G Eskin ${ }^{1,4}, \mathrm{~K}$ Pericleous ${ }^{3}$ \\ ${ }^{1}$ Brunel Centre for Advanced Solidification Technology (BCAST), Brunel University, Uxbridge, Middlesex, UB8 3PH, UK \\ ${ }^{2}$ University of Oxford, Department of Materials, Parks Road, Oxford OX1 3PH, UK \\ ${ }^{3}$ Computational Science and Engineering Group, University of Greenwich, London, SE10 9LS, UK \\ ${ }^{4}$ Tomsk State University, Tomsk, 634050 Russia
}

Keywords: cavitation, light-metal alloys, ultrasonic melt processing

\begin{abstract}
Ultrasonic cavitation treatment of melt significantly improves the downstream properties and quality of conventional and advanced metallic materials. However, the transfer of this technology to treating large melt volumes has been hindered by a lack of fundamental knowledge, allowing for the ultrasonic processing in the melt flow. In this study, we present the results of experimental validation of an advanced numerical model applied to the acoustic cavitation treatment of liquid aluminum during continuous flow [1]. This was achieved by using a calibrated high-temperature cavitometer. The acoustic spectrum was analyzed at various points across the launder while acoustic pressures were calculated at the frequencies of interest.
\end{abstract}

\section{Introduction}

It is recognized that ultrasonic treatment of metal alloys is a powerful, environment friendly, and cost effective process [2-4]. Liquid melt is subjected to high-intensity acoustic waves, resulting in important non-linear effects in liquids, i.e., cavitation and acoustic streaming. The dynamic behaviour of the cavitation bubbles has been proven effective and promising in degassing and structure refining of metallic melts as shown and reviewed in [4]. Cavitation is the formation, growth, oscillation, collapse, and implosion of bubbles in liquids [5]. In the vicinity of collapsing bubbles, extreme temperatures $(>10000 \mathrm{~K})$ [6], pressures $(>400$ $\mathrm{MPa})[6,7]$, and cooling rates $\left(>10^{11} \mathrm{~K} / \mathrm{s}\right)$ [8] occur. To enable cavitation, a sufficient amount of acoustic energy should be introduced in the melt to set up a pressure variation that initiates bubbles formation. Typically a peak-to-peak amplitude of about $10 \mu \mathrm{m}$ at $20 \mathrm{kHz}$, corresponding to acoustic pressures of greater than $0.5 \mathrm{MPa}$, is sufficient for cavitation inception in liquid aluminum $[4,9]$.

Many studies have been recently published on the effect of ultrasonic vibration on modifying and controlling the solidification structure of aluminum and magnesium alloys [9-11]. However, the fundamental mechanisms are still under scrutiny due to the experimental challenges [4, 12-13]. The prime benefit of this technology is that the ultrasonic vibrations degas the melt and refine the solidification structure of many alloys, therefore improving the quality of the end products. Cast components with refined and uniform grain structure have many advantages including significant improvement of product quality, processability, and mechanical properties [3].

A key feature of most advanced large scale casting processes is that they are performed in continuous or semi-continuous mode. Thus, to transfer and establish this promising technology into an industrial process routine, laboratory scale experiments should change emphasis from conventional static batch treatment to processing in continuous liquid flow. This brings up an important point of choosing appropriate parameters for evaluation of cavitation intensity, and thus of determining the spread of the cavitation activity in a moving volume flow. The main hypothesis is that the liquid processing occurs by passing unit volumes of the treated liquid through the active region where cavitation bubbles are formed and collapse, with acoustic and secondary flows acting as mixing and transporting means. Therefore, the dimensions and geometry of cavitation zone and the flow pattern of the melt play an important role in improving treatment efficiency. Ideally, they should be adjusted in such a way that all the metal passes through the cavitation zone. To the best of our knowledge, experimental validation and proper discussion on these issues are lacking in the scientific literature and further research is essential to reveal the conditions of a more controllable and efficient ultrasonic processing regime in different alloying systems and in large moving volumes of liquid melts, paving the way to extensive industrial use.

This study moves towards that direction as it focuses on the cavitation processing efficiency, obtained by measuring acoustic pressures in a continuous flow. A specially modified launder with baffles at specific points is used. Experiments in this part of the study are performed only in water, as water and aluminum share similar fluid properties and results obtained are easily scalable $[14,15]$. The effect of baffles separation on the pressure field is also determined. The intensity of the cavitation zone and the extent of the cavitation region in the upstream and downstream regions of the moving flow are directly measured using a novel high-temperature calibrated cavitometer [16].

\section{Methodology}

In the current study, characterization of the cavitation intensity and the corresponding acoustic pressure fields in water was conducted using an experimental set-up schematically shown in Figure 1. Ultrasonic excitation was achieved by a $500 \mathrm{~W}$ piezoelectric generator (Sonics/UK) which oscillates in a nominal fundamental frequency at $20 \mathrm{kHz}$. Vibrations were introduced into the liquid media by vertically immersing a stepped titanium sonotrode with $20-\mathrm{mm}$ tip diameter to a depth of approximately $20 \mathrm{~mm}$ into the liquid volume. Peak-to-peak vibration amplitudes at the tip of the sonotrode were varied from $0.25 \mu \mathrm{m}$ to $30 \mu \mathrm{m}$. The sonotrode was placed in the middle of the $1000 \mathrm{~mm}$ in length launder and equidistant from both baffles. Some of the key material properties for water are listed in Table 1.

The launder is $1000 \mathrm{~mm}$ along y and $90 \mathrm{~mm}$ along $\mathrm{x}$. The bottom section is a half cylinder of radius $45 \mathrm{~mm}$. The liquid is filled up to a height of $50 \mathrm{~mm}$ from the bottom of the launder. The baffles have a thickness $8 \mathrm{~mm}$. The lower opening of the upstream baffle (baffle 1) is $20 \mathrm{~mm}$, and the height of the downstream baffle 
(baffle 2) is $40 \mathrm{~mm}$. Two cases were considered in this study with: i) moving flow at 5.5 liters per minute $\left(\mathrm{cm}^{3} / \mathrm{min}\right)$ and ii) stationary liquid $\left(0 \mathrm{~cm}^{3} / \mathrm{min}\right)$.

The distance between the baffles is fixed at $148 \mathrm{~mm}$ tailored to the sound wavelength of $74 \mathrm{~mm}$ at $20 \mathrm{kHz}$ for water. To investigate the effect of the baffles on cavitation intensity, the measurements of acoustic emissions were taken at several points as shown in Fig. 1 (indicated by the capital letters A, B, C). Acoustic pressure is also measured at 3 points in the domain $\mathrm{A}$ (below the sonotrode and inside the cavitation zone), B (in the upstream of the flow) and $\mathrm{C}$ (in the downstream of the flow), with and without moving flow across the launder. To achieve reproducible and comparable data, each test was carried out at least 3 times.

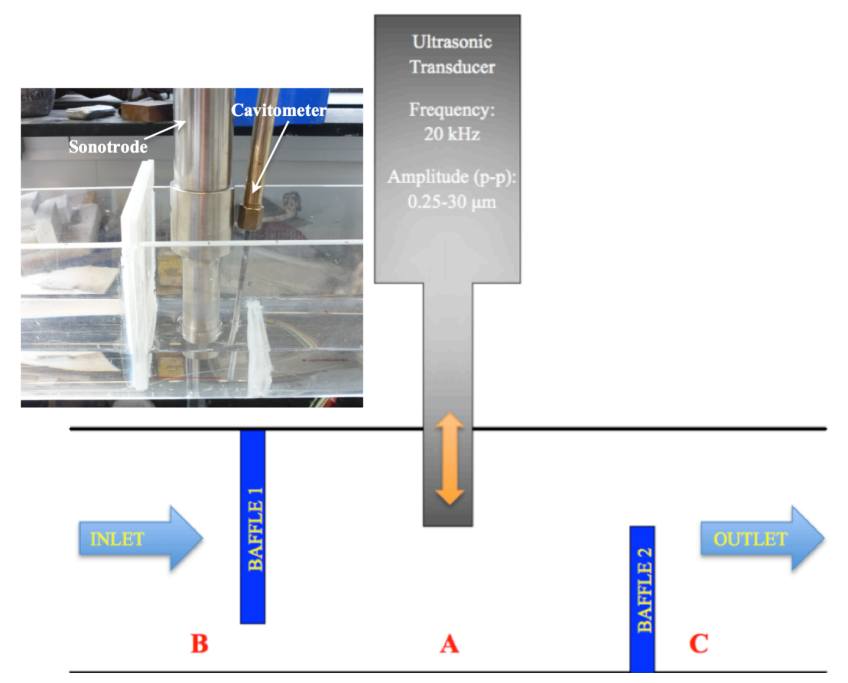

Figure 1: Schematic overview of the test arrangement with the sonotrode placed in the middle of the launder and the baffles at equal distance $(7.5 \mathrm{~cm})$ from the sonotrode in the upstream (Baffle 1) and downstream (Baffle 2) of the moving flow. Measurements performed with a calibrated cavitometer (see inset in Fig. 1) placed in the points indicated by the letters A (inside the cavitation zone), B (upstream), C (downstream).

Features of the acoustic emissions were captured and converted into meaningful acoustic pressures using an advanced calibrated cavitometer ICA-3HT (BSUIR/Belorussia) equipped with a 4-mm diameter tungsten probe, with a spatial resolution of $50 \pm 10 \mathrm{~mm}$ and a bandwidth of up to $10 \mathrm{MHz}$. The cavitometer was specifically designed to measure cavitation activity in high temperature melts and in high power ultrasonic fields, i.e. in molten metals, although it can measure equally well cavitation activity in low temperature liquids. A full account of the cavitometer design and performance can be found in [16].

Table 1: Material properties of water.

\begin{tabular}{|c|c|}
\hline Material Property & Water $\left(20^{\circ} \mathrm{C}\right)$ \\
\hline Sound speed $(\mathrm{m} / \mathrm{s})$ & 1482 \\
Density $\left(\mathrm{kg} / \mathrm{m}^{3}\right)$ & 1000 \\
Dynamic viscosity $(\mathrm{mPa} \mathrm{s})$ & 1.004 \\
\hline
\end{tabular}

The frequency spectrum was acquired by an external digital oscilloscope device, Picoscope, attached to the cavitometer. The Picoscope allowed real-time signal monitoring of the cavitometer sensor's data and ultrasonic parameters. The raw voltage signal is transformed to the frequency values via a Fast Fourier Transform. A number of 30 signal averages of the acquired signal were typically taken using a resolution bandwidth of $500 \mathrm{~Hz}$. The time for this signal acquisition was approximately $30 \times 2 \mathrm{~ms}$ (time gate) $=60 \mathrm{~ms}$. A total of 1000 wave patterns were analyzed in each of the measurement points from Fig. 1.

\section{Results}

The local cavitation phenomena in the launder for both cases can be explained based on the spectral characteristics of acoustic emissions. RMS (root mean square) acoustic pressures are shown in Figures 2 and 3 for the driving frequency $20 \mathrm{kHz}$ and at an acoustic frequency of $1 \mathrm{MHz}$ (associated with acoustic pressures exerted from the stable cavitation behavior of tiny in size bubbles, i.e. in the range of $3 \mu \mathrm{m}$, or from the transient activity of cavitation bubbles of any size) at the points of interest (A, B, C) for stationary liquid as well as for the flow.

In Figure 2, acoustic pressures in the range of the driving frequency for both of the studied cases are shown for the three different positions along the launder. In the case where the liquid volume is stationary and at low, pre-cavitation amplitudes of vibration, there is a significantly higher range of pressures in the upstream region (point B) compared with the downstream (point C). After a period of rapid increase up to the cavitation threshold a significant drop in measured acoustic pressures is observed due to the cavitation cloud shielding effect, which limits the penetration of the driving acoustic field into the liquid. Shielding is the formation of cluster of bubbles just in front of the tip of the sonotrode distracting the propagation of acoustic emissions from the incident source and the cavitation bubbles [17]. In the downstream region, the values increase and remain rather steady at higher amplitudes reaching the pressure about 50\% lower than the maximum value. The reason that acoustic pressure is significantly lower and remains fairly steady in the downstream region is possibly due to the blockage of the liquid pathway from baffle 2 where only a small portion of incident or secondary acoustic emissions escape from the upper part of the baffle reaching the cavitometer probe, as the opening is about $1 \mathrm{~cm}$ above the surface of the sonotrode tip. Finally in the area below the sonotrode, and inside the cavitation zone, acoustic pressures are one order of magnitude higher than the corresponding ones in the upstream and downstream, demonstrating the importance of the liquid volume to passing through the cavitation zone for an efficient processing.

When the moving volume is considered results are slightly different especially for the two regions near the baffles as shown in Fig. 2b. In the upstream region (point B), acoustic pressures are gradually increasing with amplitude although this time the increment lasts until $15 \mu \mathrm{m}$ displacements before a significant drop occurs. In the downstream region (point $\mathrm{C}$ ), a significant increment of the acoustic pressures is observed until a prominent pressure peak at $7.5 \mu \mathrm{m}$ displacement is reached. Cavitation intensity is then decreased with pressure values being similar or higher than that in the upstream region, depending on the amplitude of oscillations. Inside the cavitation zone (point A) pressures are similar to the stationary case, staying mainly unaffected from the flow regime.

When the flow is introduced, the initially measured downstream acoustic pressure gets significantly higher, about $40 \%$, reaching 
similar pressure values to that of the upstream region. The main hypothesis is that since the flow is much slower than the speed of sound it should not influence the acoustic propagation. Therefore, the only reasonable explanation is that the transport of cavitating bubbles towards the downstream region causes the change in acoustic pressure pattern. Bubbles migrate with flow towards the downstream end and due to their non-linear behavior or subsequent collapse, contribute further to the acoustic pressure at $20 \mathrm{kHz}$ (superimposed to the main/incident signal from the source). In addition, the shielding effect is less pronounced at the moderate amplitudes as the bubbly cloud is dispersed by the flow.
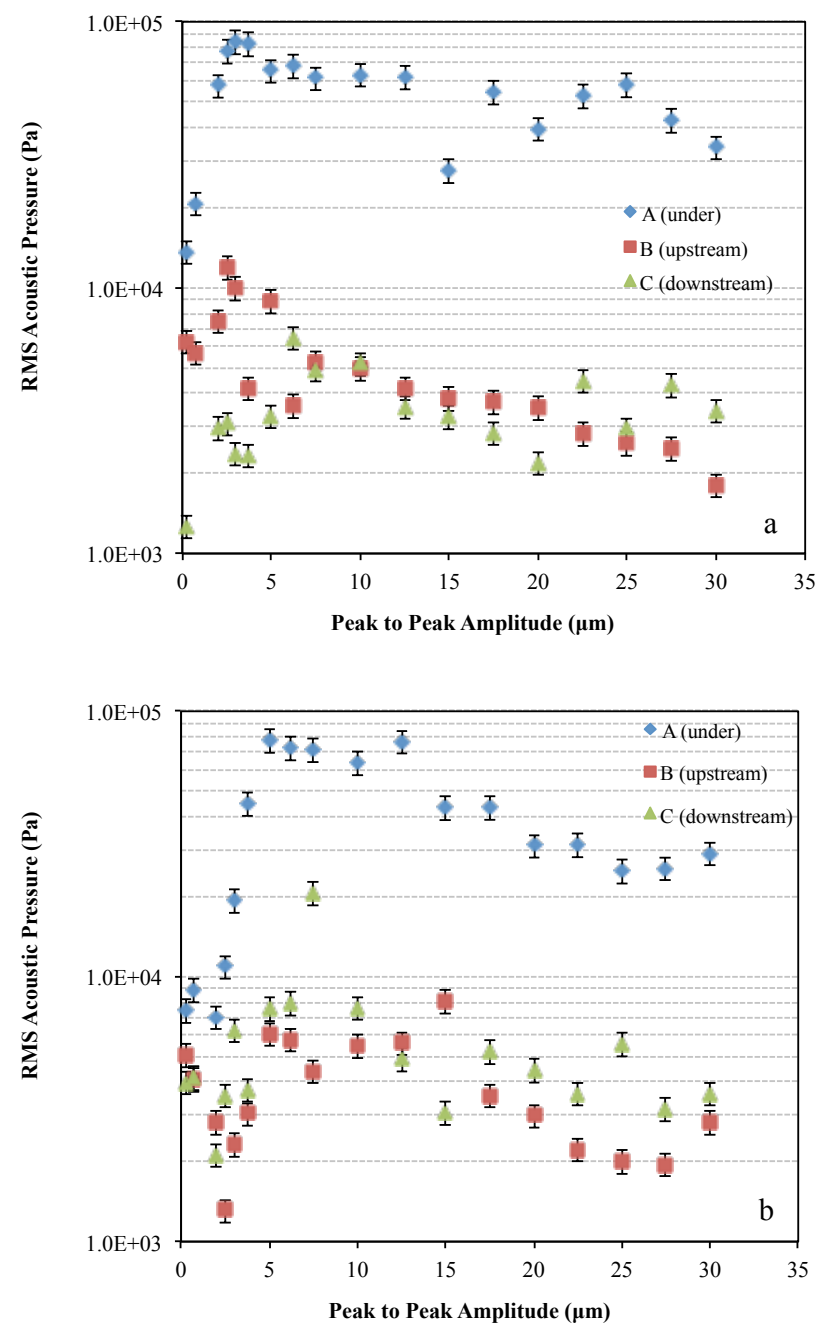

Figure 2: Variation in RMS acoustic pressures of the driving frequency $(20 \mathrm{kHz})$ as a function of the tip amplitude for three different regions across the experimental launder. Measurements were taken for a) stationary flow and b) moving flow.

Results in Figure 3 indicate that acoustic emissions from cavitation bubbles at $1 \mathrm{MHz}$ and thus the cavitation regime in general are not affected by the fluid flow. It is clear, for all the studied regions, that cavitation intensity from bubbles behavior follows the same pressure pattern regardless the flow regime. It is interesting that the upstream pressure is actually higher that the downstream, which makes the processing time of the unit volume longer and effective even before the liquid reaches the cavitation zone. This also implies that fluid flow is not necessary increases the efficiency of bubbles' transport downstream as bubbles can be also migrated with the acoustic streamers induced by the sonication process. For the migration of larger in size cavitation bubbles (larger than $150 \mu \mathrm{m}$ as the resonance size of bubbles at 20 $\mathrm{kHz}$ is $160 \mu \mathrm{m}$ ), fluid flow maybe necessary in order for these bubbles to reach areas behind baffle 2. However, for tiny cavitation bubbles in the size of a few microns, which are registered with the acoustic emissions at $1 \mathrm{MHz}$, fluid flow does not play any significant role.
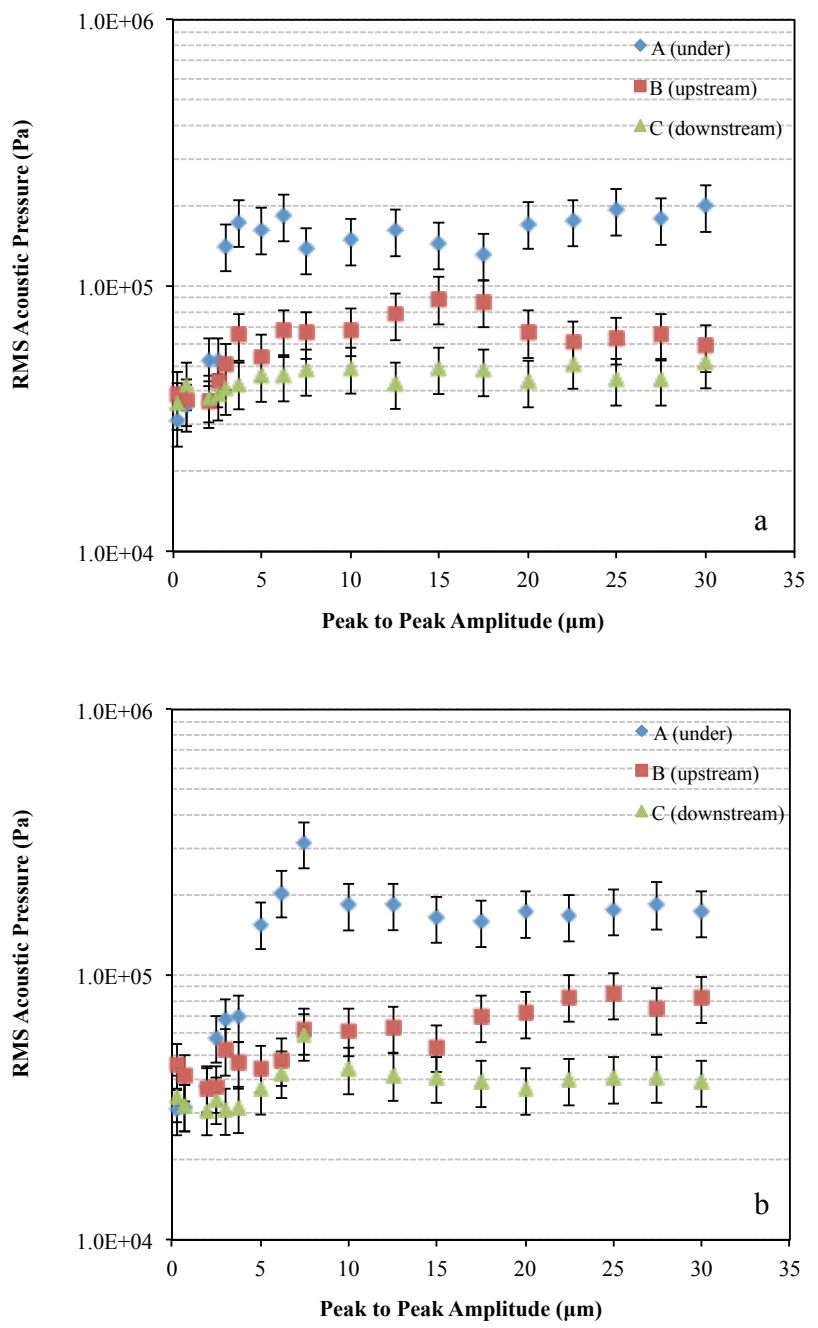

Figure 3: Variation in RMS acoustic pressures at $1 \mathrm{MHz}$ frequency (associated with transient cavitation bubble behaviour and emissions from stable oscillating cavitation bubbles in the range of $3 \mu \mathrm{m}$ ) as a function of the tip amplitude for three different regions across the experimental launder. Measurements were taken for a) stationary flow and b) moving flow.

\section{Conclusions}

The interaction between the cavitation zone and the moving flow is an important component in ultrasonic melt processing. In this study, cavitation acoustic pressures generated along a specially modified with baffles launder were measured with the use of a calibrated cavitometer. Baffles were used to slow down the flow 
passing through the cavitation zone and thus to increase the residence time of the moving flow into the cavitation zone for better treatment. Results showed that flow can enhance the pressure regime at low frequencies associated with larger in size cavitation bubbles along the studied regions and especially in the downstream region where without the flow the absence of high acoustic pressures was noticeable. When acoustic pressures at 1 $\mathrm{MHz}$, mainly associated with the stable cavitation emissions from smaller in size bubbles, are considered, the acoustic pressure pattern stayed mainly unaffected implying that the transportation of such a small in size bubbles can be easily achieved with the acoustic streamers generated from the acoustic source. The higher emissions from cavitation bubbles observed upstream suggest that the zone of active cavitation processing extends beyond the cavitation zone itself and can be managed by the flow management. The findings of this study along with the used technique are needed for eventual industrial implementation and scale up of ultrasonic processing technologies. A better control of the acoustic pressure fields and cavitation development holds the key for the optimization of casting processes.

\section{Acknowledgments}

This work is performed within the Ultramelt Project supported by the EPSRC Grants EP/K005804/1 and EP/K00588X/1.

\section{References}

[1] G.S.B. Lebon, I. Tzanakis, K. Pericleous, D. Eskin. A highorder acoustic cavitation model for the treatment of a moving liquid metal volume, Proc. CFD Modeling and Simulation in Materials Processing, Wiley: TMS, 2016 (in press)

[2] J. Campbell "Effects of vibration during solidification" Intern. Met. Rev. 26 (1981) 71-108.

[3] O. V. Abramov "Ultrasound in Liquid and Solid Metals" Boca Raton: CRC Press, 1994.

[4] G.I. Eskin, D.G. Eskin "Ultrasonic treatment of light alloy melts" Second Edition, Series: Advances in Metallic Alloys, CRC Press, 2014

[5] T. G. Leighton "The acoustic bubble", Ed. Academic Press, London, 1994.

[6] D. J. Flannigan, K. S. Suslick "Plasma formation and temperature measurement during single-bubble cavitation" Nature 434 (2005) 52-55

[7] I. Tzanakis, D.G. Eskin, A. Georgoulas, D. Fytanidis "Incubation pit analysis and calculation of the hydrodynamic impact pressure from the implosion of an acoustic cavitation bubble." Ultrasonics Sonochemistry 21 (2014) 866-878.

[8] A. Gedanken "Using sonochemistry for the fabrication of nanomaterials" Ultrasonics Sonochemistry 11 (2004) 47-55

[9] S. Komarov, K. Oda, Y. Ishiwata, N. Dezhkunov "Characterization of acoustic cavitation in water and molten aluminium alloy" Ultrasonics Sonochemistry, 20 (2013) 754-761

[10] W. Khalifa, Y. Tsunekawa, M. Okumiya, "Effect of ultrasonic treatment on the Fe-intermetallic phases in ADC12 die cast alloy" J. Mater. Process. Technol. 210 (2010) 2178

[11] T.V. Atamanenko, D. G. Eskin, L. Zhang, L. Katgerman "Criteria of Grain Refinement Induced by Ultrasonic Melt
Treatment of Aluminum Alloys Containing $\mathrm{Zr}$ and Ti" Metall. Mater. Trans. A, 41A (2010) 2056-2066

[12] E.A. Heidemann "Metallurgical effects of ultrasonic waves" J. Acoust. Soc. Am., 26 (1954) 831-842.

[13] K.S. Suslick, Y. Davidenko, M.M. Fang et al. "Acoustic cavitation and its chemical consequences" Phil. Trans. R. Soc. Lond. A, 1999, vol. 357, pp. 335-353

[14] D. Xu, W.K. Jones, Jr., J.W. Evans "The use of PIV in the physical modelling of flow in EM or DC casting of aluminium: Part I. Development of the physical model" Metall. Mater. Trans. B, 29B (1998) 1281-1288

[15] I. Tzanakis, G.S.B. Lebon, D.G. Eskin, K.A. Pericleous "Characterisation of the ultrasonic acoustic spectrum and pressure field in aluminium melt with an advanced cavitometer", J. Mater. Process. Technol. (in press)

[16] I. Tzanakis, M. Hodnett, B. Lebon, N. Dezhkunov, D.G. Eskin "Calibration and performance assessment of an innovative high-temperature cavitometer" Sensors and Actuators A: Physical (Under Review)

[17] L.D. Rozenberg, in "Powerful Ultrasonic Fields", Part VI Cavitation Region, Nauka, Moscow, 1968, pp. 221-266 